\title{
HypoparaNet: A Database of Chronic Hypoparathyroidism Based on Expert Medical-Surgical Centers in Italy
}

\author{
Gemma Marcucci ${ }^{1} \cdot$ Luisella Cianferotti $^{1} \cdot$ Simone Parri ${ }^{1} \cdot$ Paola Altieri ${ }^{2} \cdot{\text { Emanuela } \text { Arvat }^{3} \cdot \text { Salvatore Benvenga }}^{4}$. \\ Corrado Betterle ${ }^{5}$. Marta Bondanelli ${ }^{6} \cdot$ Marco Boscaro $^{5}$. Valentina Camozzi ${ }^{5}$. Grazia Maria Centaro ${ }^{7}$. \\ Filomena Cetani ${ }^{8} \cdot$ Iacopo Chiodini $^{9} \cdot$ Anna Ciampolillo $^{10}$. Annamaria Colao ${ }^{11}$. Sabrina Corbetta ${ }^{12}$. \\ Maria Laura De Feo ${ }^{13}$. Ettore degli Uberti ${ }^{6}$. Antongiulio Faggiano ${ }^{14}$. Rachele Fornari ${ }^{15}$. Achille Lucio Gaspari ${ }^{16}$. \\ Francesco Giorgino ${ }^{10} \cdot$ Valeria Giuliani $^{13} \cdot$ Maurizio lacobone $^{17} \cdot$ Nadia Innaro $^{18}$. Olga Lamacchia ${ }^{19} \cdot$ Andrea Lenzi $^{15}$. \\ Giovanna Mantovani ${ }^{9}$. Claudio Marcocci ${ }^{20}$. Laura Masi $^{21}$ - Silvia Migliaccio ${ }^{22}$. Serena Palmieri ${ }^{9} \cdot$ Renato Pasquali $^{2}$. \\ Giuliano Perigli ${ }^{23}$. Valentina Piccini ${ }^{13}$. Elisabetta Romagnoli ${ }^{15}$. Rosaria Maddalena Ruggeri ${ }^{4} \cdot$ Francesco Rulli $^{24}$. \\ Maria Teresa Samà ${ }^{3}$. Giuseppe Tomaino ${ }^{17}$. Francesco Trimarchi ${ }^{25} \cdot$ Maria Chiara Zatelli $^{6} \cdot$ Maria Luisa Brandi $^{1}$ (1)
}

Received: 10 December 2017 / Accepted: 2 March 2018 / Published online: 6 March 2018

(c) Springer Science+Business Media, LLC, part of Springer Nature 2018

\begin{abstract}
Hypoparathyroidism is a rare disease characterized by low serum calcium levels and absent or deficient parathyroid hormone level. Regarding the epidemiology of chronic hypoparathyroidism, there are limited data in Italy and worldwide. Therefore, the purpose of this study was to build a unique database of patients with chronic hypoparathyroidism, derived from the databases of 16 referral centers for endocrinological diseases, affiliated with the Italian Society of Endocrinology, and four centers for endocrine surgery with expertise in hypoparathyroidism, to conduct an epidemiological analysis of chronic hypoparathyroidism in Italy. The study was approved by the Institutional Review Board. A total of 537 patients with chronic hypoparathyroidism were identified. The leading etiology was represented by postsurgical hypoparathyroidism (67.6\%), followed by idiopathic hypoparathyroidism (14.6\%), syndromic forms of genetic hypoparathyroidism (11\%), forms of defective PTH action (5.2\%), non-syndromic forms of genetic hypoparathyroidism ( $0.9 \%)$, and, finally, other forms of acquired hypoparathyroidism, due to infiltrative diseases, copper or iron overload, or ionizing radiation exposure $(0.7 \%)$. This study represents one of the first large-scale epidemiological assessments of chronic hypoparathyroidism based on data collected at medical and/or surgical centers with expertise in hypoparathyroidism in Italy. Although the study presents some limitations, it introduces the possibility of a large-scale national survey, with the final aim of defining not only the prevalence of chronic hypoparathyroidism in Italy, but also standards for clinical and therapeutic approaches.
\end{abstract}

Keywords Epidemiology $\cdot$ Parathyroid hormone $\cdot$ Hypoparathyroidism $\cdot$ Therapy $\cdot$ Thyroidectomy

\section{Background}

Hypoparathyroidism is a rare endocrine disorder characterized by hypocalcemia and hyperphosphatemia, due to absent or inappropriately low serum parathyroid hormone (PTH) levels. It may be caused by acquired and hereditary

Electronic supplementary material The online version of this article (https://doi.org/10.1007/s00223-018-0411-7) contains supplementary material, which is available to authorized users.

Maria Luisa Brandi

marialuisa.brandi@unifi.it

Extended author information available on the last page of the article conditions [1-3]. Pseudohypoparathyroidism (PHP) is a less common disorder due to target organ resistance to PTH, characterized by similar mineral biochemical abnormalities, but with elevated circulating concentrations of PTH [1].

Data in literature show that chronic hypoparathyroidism results from a surgical procedure in approximately $75 \%$ of patients and from genetic, autoimmune, or idiopathic etiologies in the remaining cases [1]. Chronic postsurgical hypoparathyroidism is generally defined as permanent when PTH is insufficient to maintain normocalcemia 6 months after surgery $[2,4]$. Post-thyroid surgery hypoparathyroidism has a prevalence of $0.9-1.6 \%$ when thyroidectomy is performed by experienced endocrine surgeons, but it is as high 
as $6.6 \%$ when thyroidectomy is performed by general surgeons [2, 5-7]. In the case of surgery for substernal goiter, head or neck malignancies with involvement of the anterior neck structures, or Graves' disease, a higher risk of postoperative hypoparathyroidism has been demonstrated [1]. The second most common cause of adult hypoparathyroidism is autoimmunity, either restricted to the parathyroids or encompassing other endocrine glands, such as autoimmune polyglandular syndrome type 1-3-4 (APS-1-3-4) or, rarely, APS-2 [2]. Other forms of genetic hypoparathyroidism can be associated with other organ defects or occur as isolated disorder. The remaining etiologies are rare and include infiltrative disease of metastatic or mineral-overload nature (copper, iron) or damage by ionizing radiation exposure. Finally, the term "idiopathic hypoparathyroidism" is used when the underlying cause is unknown or not investigated [1].

Little data exist on the epidemiology and natural history of chronic hypoparathyroidism worldwide.

In Denmark, through registries and individual patient hospital charts, a total of 180 patients with non-surgical hypoparathyroidism were identified, among whom 123 were alive at the date of follow-up (prevalence of 2.3/100,000 inhabitants) [8]. A Danish study also identified 1849 patients with postsurgical hypoparathyroidism through the Danish National Patient Registry and a prescription database [9]. In this survey, the prevalence of postsurgical hypoparathyroidism was 22/100,000 inhabitants. Compared with controls, patients with hypoparathyroidism had an increased risk of renal complications and hospitalization due to seizures [9]. Recently, another Danish study showed that postsurgical hypoparathyroidism was associated with an increased risk of depression and other types of neuropsychiatric diseases, as well as infections, whereas patients seemed to be protected against fractures at the upper extremities and gastrointestinal malignancies, compared with controls [10].

In the USA, the prevalence of hypoparathyroidism was estimated on the basis of the diagnosis of hypoparathyroidism in a large private health plan database over 12 months and projected to the USA insured population [11]. Incidence was also calculated from the same database by determining the proportion of total neck surgeries resulting in either transient ( $\leq 6$ months) or chronic ( $>6$ months) hypoparathyroidism. The surgical-based incidence approach yielded 117,342 relevant surgeries resulting in 8901 cases over 12 months. The diagnosis-based prevalence approach estimated 58,793 insured patients with chronic hypoparathyroidism in the USA over the total number of insured patients (7.8 million patients) [11].

Recently, Astor MC et al. conducted an epidemiologic survey on hypoparathyroidism in Norway [12]. Patients with hypoparathyroidism and 22q11 deletion syndrome were identified with electronic hospital registries. Five hundred twenty-two patients were identified, with an estimated overall prevalence of 102 per million divided among postsurgical hypoparathyroidism (64 per million), and non-surgical hypoparathyroidism (30 per million). Most patients were treated with vitamin D and calcium, and only ten patients received recombinant human PTH (rhPTH). Patients with hypoparathyroidism had a significantly worse score than the normal population regarding Short Form 36 and Hospital Anxiety and Depression scale [12].

In Italy, only a recent retrospective, register-based study, although mainly focused on hospitalized postsurgical cases, was performed by analyzing records of hospital discharge over an 8-year period [13]. In this analysis, the mean prevalence of hypoparathyroidism among inpatients was 5.3/100,000 inhabitants per year [13]. No other analyses have been conducted in our country so far.

Current treatment options for management of chronic hypoparathyroidism are oral calcium and vitamin D (including its metabolites and analogs) supplements, and thiazide diuretics to reduce urinary calcium excretion [14]. Recently, two guidelines identified the main aims of treatment: to maintain serum calcium levels in the low normal range, or concentration slightly below normal (i.e., no more than $0.5 \mathrm{mg} / \mathrm{dl}$ below normal); to prevent signs and symptoms of hypocalcemia; to avoid hypercalcemia; to maintain serum phosphate within the normal range, and total calcium-phosphate product below $4.4 \mathrm{mmol}^{2} / \mathrm{l}^{2}\left(55 \mathrm{mg}^{2} / \mathrm{dl}^{2}\right)$; to avoid hypercalciuria; and to avoid renal (nephrocalcinosis/ nephrolithiasis) and other extraskeletal calcifications [14, 15]. However, currently, we do not know what percentage of patients are adequately treated with standard treatment in accordance with these guidelines.

Over the past two decades, studies of recombinant human (rh)PTH (1-34) and (1-84) have ushered in a new era for the management of this disease, with an improvement of calcium homeostasis, particularly for patients who require large amounts of calcium and active vitamin D, and are not adequately controlled by conventional therapy [14]. To date, in the USA, the Food and Drug Administration (FDA) approved rhPTH (1-84) for the management of selected patients with chronic hypoparathyroidism in 2015 [16]. Then, in Europe, in February 2017, the European Medicines Agency (EMA) recommended granting a conditional marketing authorization in the European Union (EU) for Natpar [rhPTH (1-84)]. One guideline recommends taking into consideration rhPTH (1-84) therapy in patients with chronic hypoparathyroidism of any etiology, except autosomal-dominant hypocalcemia (ADH), which cannot be controlled well with calcium and active vitamin $\mathrm{D}$, in particular in case of: variable and inconstant control of the serum calcium with frequent episodes of hypo- and hypercalcemia; oral calcium/vitamin D medications required to control the serum calcium or symptoms that exceed $2.5 \mathrm{~g}$ of calcium or $>1.5 \mu \mathrm{g}$ of active vitamin $\mathrm{D}$; hypercalciuria, 
renal stones, nephrocalcinosis, stone risk, or reduced creatinine clearance or eGFR ( $<60 \mathrm{ml} / \mathrm{min})$; hyperphosphatemia and/or calcium-phosphate product that exceeds $55 \mathrm{mg}^{2} \mathrm{dl}^{2}$ $\left(4.4 \mathrm{mmol}^{2} \mathrm{l}^{2}\right)$; gastrointestinal tract disorder that is associated with malabsorption; and, finally, reduced quality of life [14]. In most cases, rhPTH therapy will not be needed, but exactly how many patients could benefit from this new treatment is still unclear in Italy, as well as in Europe.

Given that there is little epidemiological data regarding this endocrinological disease, and that a new treatment with rhPTH (1-84) will soon be available, the need for epidemiological data in Italy on chronic hypoparathyroidism is urgently felt. Therefore, we initiated an Italian survey encompassing both endocrinology and endocrine surgery centers with a long history of experience in the management of hypoparathyroidism. The survey was endorsed by the Italian Society of Endocrinology [Società Italiana di Endocrinologia (SIE)], and was conducted throughout the national territory as a retrospective observational study of chronic hypoparathyroidism, with the final goal of obtaining information on the epidemiology, and main clinical and therapeutic aspects of chronic hypoparathyroidism in the Italian referral centers involved.

\section{Methods}

A retrospective observational analysis of data derived from clinical databases on chronic hypoparathyroidism by 16 Italian centers for endocrinology, affiliated with the Italian Society of Endocrinology (SIE), and 4 Italian centers for endocrine surgery with expertise in hypoparathyroidism was conducted from March 2014 to September 2015. The centers involved in the project are distributed homogeneously in the Italian territory (six centers in the north, seven in the center and seven in the south). The study was coordinated by the Unit of Bone and Mineral Metabolism Diseases of the University Hospital of Florence, Florence, Italy.

A database for chronic hypoparathyroidism was created, and each center retrospectively collected requested data. All data inserted in the database derive from the baseline evaluation reported in clinical records of each center. Then, all data were collected and analyzed by the project coordinating center. This database included all subjects affected by chronic hypoparathyroidism of all ages and races, with a baseline evaluation at each center varying between 1980 and 2015. Some patients with chronic hypoparathyroidism could not be included in this analysis because they were not evaluated by specialized centers. The cases of postsurgical chronic hypoparathyroidism were diagnosed at least 6 months after neck surgery. All patients were undergoing treatment.
All available data concerning demographic data (sex, age), age of onset of the disease, year of the first assessment (baseline evaluation) at the specialist center, type of chronic hypoparathyroidism, description of type of thyroid/parathyroid surgery (in case of postsurgical hypoparathyroidism), genetic tests, clinical signs and symptoms of hypoparathyroidism at onset of disease and at baseline evaluation, biochemical exams of bone metabolism, instrumental exams performed, and type of therapy at the baseline evaluation performed at each referral center were collected and analyzed. Data on patients with pseudohypoparathyroidism (PHP) were analyzed separately from the other forms of chronic hypoparathyroidism.

All clinical data were collected anonymously, using the initials of the name and date of birth of each patient, using an electronic excel sheet. Table 1 shows the clinical questionnaire used to collect data derived from the medical records.

Analysis of frequencies and descriptive statistics were performed using the IBM Statistical Package for Social Sciences (SPSS 20.0) for Windows (IBM, Armonk, NY, USA).

The study was approved by the Institutional Review Board (Comitato Etico Area Vasta Centro, AUOC, Florence, Italy) [number: 10641_oss]. Consent for the publication of the data was obtained. Informed consent was collected in accordance with General Authorization to Process Personal Data for Scientific Research Purposes (Authorization no. 9/2013, The Italian Data Protection Authority).

\section{Results}

A total of 537 subjects with chronic hypoparathyroidism were identified in the 20 Italian endocrinology and endocrine surgery centers participating in this study (Fig. 1). Among these, 28 cases were subjects affected by PHP. Of the patients identified in the database with chronic hypoparathyroidism, except patients with PHP, 399 (78.4\%) were female and $110(21.6 \%)$ male. The mean age of these patients at baseline evaluation was $48 \pm 19.68(\mathrm{SD})$ years, $50 \pm 18.85$ years for female and $43 \pm 21.80$ years for male, 48 subjects $<18$ years $(9.4 \%)$, and $461 \geq 18$ years $(90.6 \%)$.

There was a predominance of the female gender, less evident, also in patients with PHP [(16 female $(57.1 \%)$ and 12 male (42.9\%)], while the mean age of these patients at baseline evaluation was lower [ $24 \pm 12.99$ (SD) years, 23 years \pm 13.25 for female, and $25 \pm 13.17$ years for male, 11 subjects $<18$ years $(39.3 \%)$, and $17 \geq 18$ years $(60.7 \%)$ ].

The mean time span between the onset of chronic hypoparathyroidism in childhood and the first evaluation at each specialist center was 5.7 years, and between the onset of hypoparathyroidism in adulthood was 6.5 years. In case of PHP, it was respectively 2 and 8 years. 
Table 1 This table shows the clinical questionnaire used to data collection

Clinical database

Personal data

Diagnosis

Symptoms of onset

Data on genetic analysis

Symptoms-Basal Evaluation

Basal instrumental evaluation

Blood - urinary tests—-basal evaluation

Chronic treatment—basal evaluation

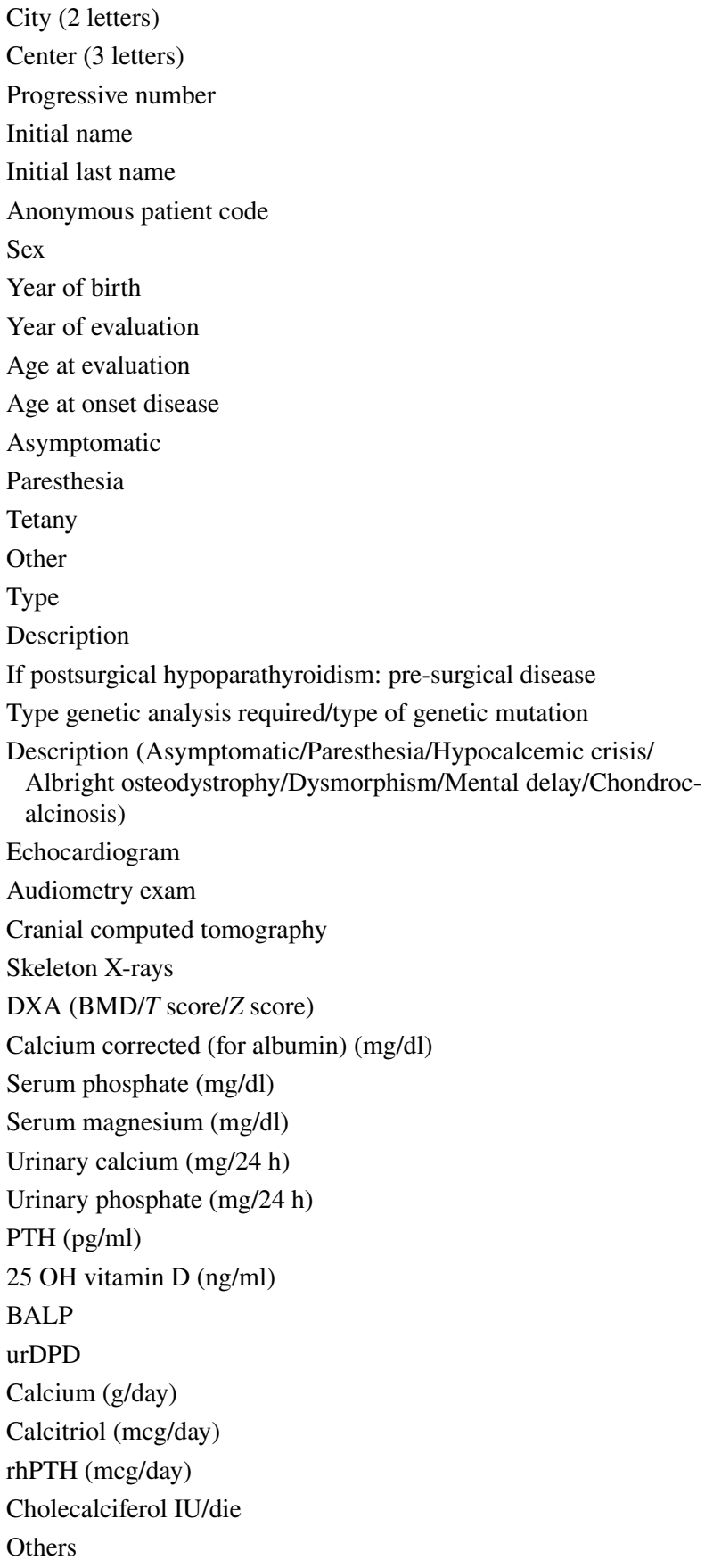

Center (3 letters)

Progressive number

Initial name

Initial last name

Anonymous patient code

Sex

Year of birth

Year of evaluation

Age at evaluation

Age at onset disease

Asymptomatic

Paresthesia

Tetany

Other

If postsurgical hypoparathyroidism: pre-surgical disease

Type genetic analysis required/type of genetic mutation

Description (Asymptomatic/Paresthesia/Hypocalcemic crisis/ Albright osteodystrophy/Dysmorphism/Mental delay/Chondroc-

Cranial computed tomography

Skeleton X-rays

Calcium corrected (for albumin) (mg/dl)

Serum phosphate $(\mathrm{mg} / \mathrm{dl})$

Urinary calcium (mg/24 h)

Urinary phosphate $(\mathrm{mg} / 24 \mathrm{~h})$

PTH $(\mathrm{pg} / \mathrm{ml})$

BALP

Calcitriol (mcg/day)

hPTH (mcg/day)

Others

PTH Parathyroid hormone, $25 \mathrm{OH}$ vitamin D 25 hydroxyvitamin D, BALP bone-specific alkaline phosphatase, urDPD urine Deoxypyridinoline, $r h P T H$ recombinant hormone PTH, $D X A$ Dual-energy X-ray absorptiometry, $B M D$ bone mineral density

\section{Etiology}

The largest percentage of cases consisted of postsurgical hypoparathyroidism $(67.6 \%)$, followed by idiopathic hypoparathyroidism (14.6\%), syndromic forms of genetic hypoparathyroidism (11\%), forms of defective PTH action
(5.2\%), non-syndromic forms of genetic hypoparathyroidism $(0.9 \%)$, and, finally, other forms of acquired hypoparathyroidism, due to infiltrative diseases, copper or iron overload, or ionizing radiation exposure $(0.7 \%)$ (Fig. 2). The patients with chronic hypoparathyroidism from iron overload due to thalassemia were probably not referred to the network of 


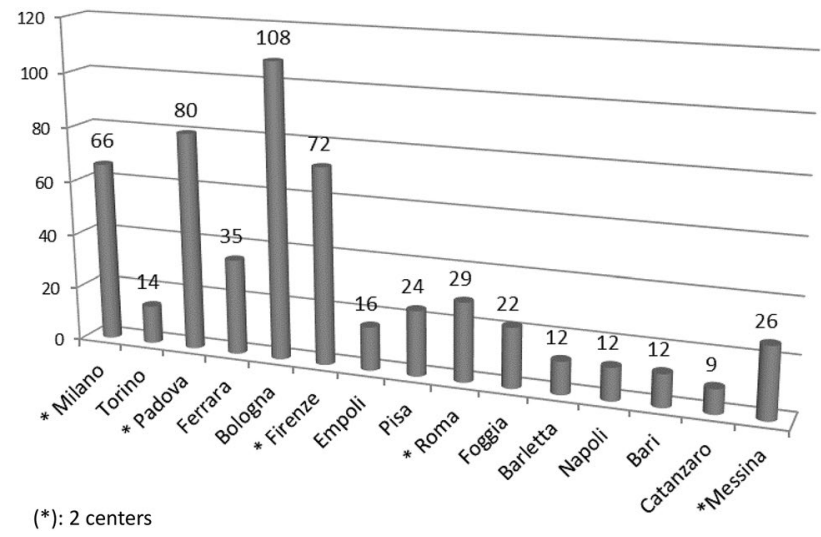

Fig. 1 Number of cases with chronic hypoparathyroidism, collected by all centers involved in the study

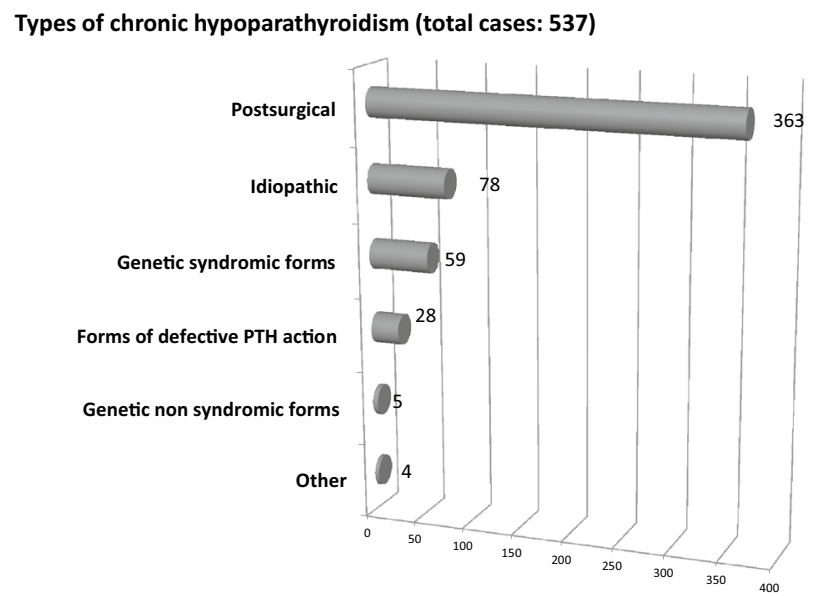

Fig. 2 The frequency of all main types of chronic hypoparathyroidism described

endocrine centers and, therefore, the number of these cases could be underestimated.

Most of the cases of postsurgical hypoparathyroidism $(87.5 \%)$ occurred after a total thyroidectomy, and $7.1 \%$ of cases after a parathyroidectomy with removal of multiple parathyroid glands. Partial thyroidectomy accounted for $3.7 \%$ of cases, and parathyroidectomy with removal of a single parathyroid gland accounted for $1.7 \%$ (after bilateral exploration) (Fig. 3). The most frequent pre-surgical pathologies were thyroid adenoma or goiter or microcarcinoma (56\%), followed by other thyroid cancers (35.6\%), familial primary hyperparathyroidism (f-PHPT) (5\%), and sporadic primary hyperparathyroidism (s-PHPT) (3.4\%) (Table 2).

Tables 3 and 4 describe the frequency analysis of the number of patients, under and over/equal to 18 years of age, suffering from various types of chronic hypoparathyroidism. These data show that most causes of chronic hypoparathyroidism under 18 years of age were syndromic forms of

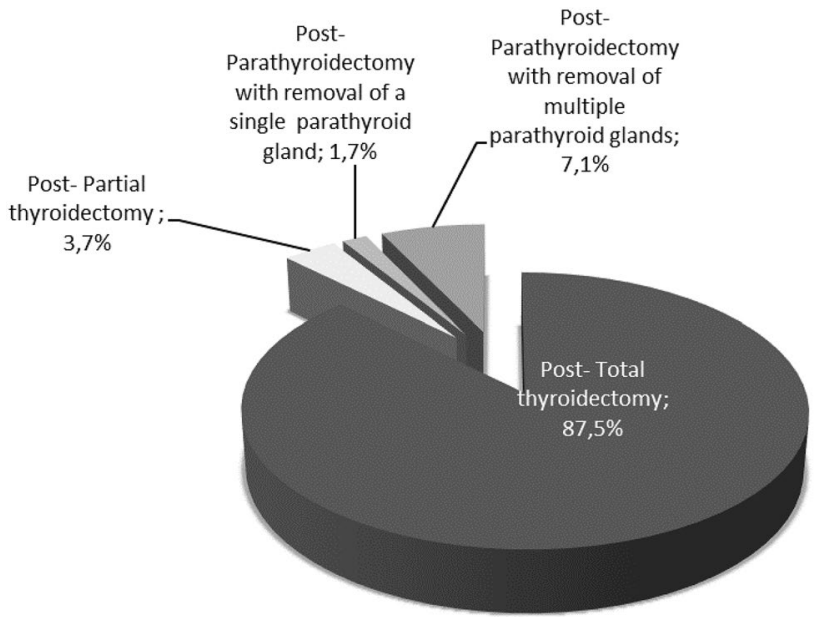

Fig. 3 The percentages of all types of surgical procedures, prior to the onset of postsurgical hypoparathyroidism

Table 2 Frequency analysis of pre-surgical pathologies of cases with postsurgical hypoparathyroidism

\begin{tabular}{lll}
\hline Pre-surgical pathologies & Frequency & Percentage \\
\hline $\begin{array}{l}\text { Thyroid adenoma/goiter/micro- } \\
\text { carcinoma }\end{array}$ & 200 & 56.0 \\
Thyroid cancer & 127 & 35.6 \\
f-PHPT & 18 & 5.0 \\
s-PHPT & 12 & 3.4 \\
Total & 357 & 100 \\
Missing data & 6 & - \\
Grand Total & 363 & \\
\hline
\end{tabular}

$f$-PHPT familial primary hyperparathyroidism, $s$ - $P H P T$ sporadic primary hyperparathyroidism

genetic hypoparathyroidism followed by end organ resistance to PTH action, and neck surgery. Conversely, most causes of chronic hypoparathyroidism described over/equal to 18 years of age were neck surgery, followed by syndromic forms of genetic hypoparathyroidism, by end organ resistance to PTH action, and, finally, non-syndromic forms of genetic hypoparathyroidism.

At onset of diseases, 61 cases (13.5\% of 450$)$ over/equal to 18 years and 17 cases (19.5\% of 87) under 18 years were classified as idiopathic, while only 3 cases $(0.6 \%$ of 450$)$ over/ equal to 18 years and 1 case $(1.1 \%$ of 87$)$ under 18 years had acquired forms from other causes, such as metastatic disease copper or iron overload, or ionizing radiation exposure.

\section{Symptomatology}

The clinical features at onset and at baseline evaluation of all forms of hypoparathyroidism are shown in Figs. 4, 5 and Tables S1, S2 (Supplementary files). 
Table 3 Frequency analysis of main types of chronic hypoparathyroidism

\begin{tabular}{|c|c|c|c|c|c|c|c|c|}
\hline Main types of chronic hypoparathyroidism & $\begin{array}{l}\text { Under } 18 \text { at } \\
\text { onset of } \\
\text { disease }\end{array}$ & $\begin{array}{l}\text { Over/equal to } \\
18 \text { at onset of } \\
\text { disease }\end{array}$ & $N^{*}$ & Percentage & Under $18 \mathrm{BSL}$ & $\begin{array}{l}\text { Over/equal } \\
\text { to } 18 \mathrm{BSL}\end{array}$ & $N^{*}$ & Percentage \\
\hline Postsurgical forms & 11 & 352 & 363 & 67.6 & 2 & 361 & 363 & 67.6 \\
\hline Idiopathic forms & 17 & 61 & 78 & 14.6 & 5 & 73 & 78 & 14.6 \\
\hline Genetic syndromic forms & 43 & 16 & 59 & 11.0 & 40 & 19 & 59 & 11.0 \\
\hline Forms of defective PTH action & 14 & 14 & 28 & 5.2 & 11 & 17 & 28 & 5.2 \\
\hline Genetic non-syndromic forms & 1 & 4 & 5 & 0.9 & 1 & 4 & 5 & 0.9 \\
\hline Other forms & 1 & 3 & 4 & 0.7 & 0 & 4 & 4 & 0.7 \\
\hline Total & 87 & 450 & 537 & 100 & 59 & 478 & 537 & 100 \\
\hline
\end{tabular}

$B S L$ at baseline evaluation of disease

$* N$ number of subjects

Under 18 years, the most common clinical findings at onset of disease were tetany, followed by paresthesias, but in $32.6 \%$ the patients were asymptomatic. On the other hand, over 18 years, the most common clinical findings at onset of disease were paresthesias, followed by tetany; however, in $32.2 \%$ the patients were asymptomatic.

At baseline evaluation, regardless of the center and age group, almost half of patients were asymptomatic, and half were symptomatic. Among the symptomatic patients, the older group (age equal to or over 18 years) had a twofold greater prevalence of paresthesias (36 vs. $17 \%$ ), whereas episodes of tetany were described in $15 \%$ of patients with age under 18 years and $10.1 \%$ in patients with age equal to or over 18 years.

Moreover, regarding the other clinical manifestations, 2 of 5 cases of DiGeorge syndrome presented dysmorphic features, and among 26 cases of PHP, 11 showed Albright's osteodystrophy (all cases of PHP type 1a), and 8 chondrocalcinosis (of these, 7 cases of PHP type 1a and 1 of PHP type 1b).

\section{Biochemical Data}

Biochemical exams of mineral metabolism, such as serum calcium, serum phosphate, and $24 \mathrm{~h}$ urinary calcium, collected at baseline evaluation at each center, often showed values above or below the reference range, for all types of chronic hypoparathyroidism.

In the group of patients with chronic hypoparathyroidism (total number: 509), the serum level of corrected calcium for albumin was available for $90.2 \%$ of patients [number of patients observed $(N)$ : 459]; among these, only $36.6 \%$ resulted within range $(8.5-10.1 \mathrm{mg} / \mathrm{dl}), 18.9 \%$ were between 8.0 and $8.5 \mathrm{mg} / \mathrm{dl}, 62.7 \%$ were under $8.5 \mathrm{mg} / \mathrm{dl}$, and $0.7 \%$ were over $10.1 \mathrm{mg} / \mathrm{dl}$. Moreover, 93 (20.3\%) had serum calcium levels under $8 \mathrm{mg} / \mathrm{dl}$ associated with symptoms of hypocalcemia (tingling or paraesthesia).
In patients affected by PHP (total number: 28), the serum level of corrected calcium for albumin was available for $78.6 \%$ of patients $(N: 22)$; among these, only $13.6 \%$ resulted within the range between 8.0 and $8.5 \mathrm{mg} / \mathrm{dl}$, under $8.5 \mathrm{mg} /$ $\mathrm{dl}$ in $86.4 \%$, over $10.1 \mathrm{mg} / \mathrm{dl}$ in $0 \%$, and a similar percentage $(35.7 \%)$ compared to the other group had serum calcium levels under $8 \mathrm{mg} / \mathrm{dl}$ associated with symptoms of hypocalcemia.

The level of serum phosphate in adult patients (total number: 461) with chronic hypoparathyroidism was available for $73.1 \%$ of patients $(N: 337)$; among these, $63.2 \%$ resulted within range (2.5-4.9 $\mathrm{mg} / \mathrm{dl})$, under $2.5 \mathrm{mg} / \mathrm{dl}$ in $1.8 \%$, and over $4.9 \mathrm{mg} / \mathrm{dl}$ in $35 \%$. The level of urinary phosphorus 24 $\mathrm{h}$ was available for $33.2 \%(\mathrm{~N}: 153)$; among these, $60 \%$ were within range (range $400-1300 \mathrm{mg} / 24 \mathrm{~h}$ ), under $400 \mathrm{mg} / 24 \mathrm{~h}$ in $39.9 \%$, and over $1300 \mathrm{mg} / 24 \mathrm{~h}$ in $0 \%$. The level of serum magnesium was available for $39.7 \%(N: 183)$; among these, $59 \%$ resulted within range (range $1.8-2.4 \mathrm{mg} / \mathrm{dl}$ ), under $1.8 \mathrm{mg} / \mathrm{dl}$ in $39.3 \%$, and over $2.4 \mathrm{mg} / \mathrm{dl}$ in $1.6 \%$. The calcium-phosphate product was available for $73.3 \%(N: 337)$; among these, the level was $>55 \mathrm{mg}^{2} \mathrm{dl}^{2}$ in only $0.9 \%$, and the mean value of calcium-phosphate product was $36.32 \pm 7.97$.

In PHP adult patients (total number: 17), the levels of serum phosphate was available for $82.3 \%$ of patients $(N: 14)$; among these, $57.1 \%$ resulted within range, under $2.5 \mathrm{mg} / \mathrm{dl}$ in $7.1 \%$, over $4.9 \mathrm{mg} / \mathrm{dl}$ in $35.7 \%$. The level of urinary phosphorus $24 \mathrm{~h}$ was available for $41.2 \%(N: 7)$; among these, $28.6 \%$ were within range, under $400 \mathrm{mg} / 24 \mathrm{~h}$ in $71.4 \%$, and over $1300 \mathrm{mg} / 24 \mathrm{~h}$ in $0 \%$. The level of serum magnesium was available for $41.2 \%(\mathrm{~N}: 7)$; among these, $57.1 \%$ were within range, under $1.8 \mathrm{mg} / \mathrm{dl}$ in $42.9 \%$, and over $2.4 \mathrm{mg} /$ $\mathrm{dl}$ in $0 \%$. The calcium-phosphate product was available for $82.4 \%$ (N:14); among these, $7.1 \%$ were $>55 \mathrm{Mg}^{2} \mathrm{dl}^{2}$, and the mean value of calcium-phosphate product was $35.6 \pm 13.71$.

In adult male patients (total number: 93) with chronic hypoparathyroidism, urinary calcium $24 \mathrm{~h}$ was available for $54.8 \%$ of patients $(N: 51)$; among these, in $39.2 \%$ it was 
Table 4 Frequency analysis of all sub-types of chronic hypoparathyroidism

\begin{tabular}{|c|c|c|c|c|c|c|}
\hline \multirow[t]{2}{*}{ Postsurgical hypoparathyroidism } & \multicolumn{2}{|c|}{$\begin{array}{l}\text { Under } 18 \text { at onset of } \\
\text { disease }\end{array}$} & \multicolumn{2}{|c|}{$\begin{array}{l}\text { Over/equal to } 18 \text { at onset } \\
\text { of disease }\end{array}$} & \multicolumn{2}{|l|}{ Total } \\
\hline & $N^{*}$ & $\%$ & $N$ & $\%$ & $N$ & $\%$ \\
\hline Post-total thyroidectomy & 6 & 75.0 & 303 & 87.8 & 309 & 87.5 \\
\hline Post-partial thyroidectomy & 0 & 0.0 & 13 & 3.8 & 13 & 3.7 \\
\hline $\begin{array}{l}\text { Post-parathyroidectomy with multiple removal of the parathyroid } \\
\text { glands }\end{array}$ & 2 & 25.0 & 23 & 6.7 & 25 & 7.1 \\
\hline Post-parathyroidectomy with removal of a single parathyroid gland & 0 & 0.0 & 6 & 1.7 & 6 & 1.7 \\
\hline Total & 8 & 100 & 345 & 100 & 353 & 100 \\
\hline Missing data (type description) & 3 & & 7 & & 10 & \\
\hline Grand total & 11 & & 352 & & 363 & \\
\hline \multirow[t]{2}{*}{ Non-syndromic forms of genetic hypoparathyroidism } & \multicolumn{2}{|c|}{$\begin{array}{l}\text { Under } 18 \text { at onset of } \\
\text { disease }\end{array}$} & \multicolumn{2}{|c|}{$\begin{array}{l}\text { Over/equal to } 18 \text { at onset } \\
\text { of disease }\end{array}$} & \multicolumn{2}{|l|}{ Total } \\
\hline & $N$ & $\%$ & $N$ & $\%$ & $N$ & $\%$ \\
\hline Autosomal dominant hypocalcemia with hypercalciuria type 1 & 1 & 100.0 & 2 & 66.7 & 3 & 75.0 \\
\hline Familial isolated hypoparathyroidism, autosomal dominant & 0 & 0.0 & 1 & 33.3 & 1 & 25.0 \\
\hline Total & 1 & 100 & 3 & 100 & 4 & 100 \\
\hline Missing data (type description) & 0 & & 1 & & 1 & \\
\hline Grand total & 1 & & 4 & & 5 & \\
\hline \multirow[t]{2}{*}{ Syndromic forms of genetic hypoparathyroidism } & \multicolumn{2}{|c|}{$\begin{array}{l}\text { Under } 18 \text { at onset of } \\
\text { disease }\end{array}$} & \multicolumn{2}{|c|}{$\begin{array}{l}\text { Over/equal to } 18 \text { at onset } \\
\text { of disease }\end{array}$} & \multicolumn{2}{|l|}{ Total } \\
\hline & $N$ & $\%$ & $N$ & $\%$ & $N$ & $\%$ \\
\hline Autoimmune polyglandular syndrome type $1-2$ & 39 & 90.7 & 14 & 93.3 & 53 & 91.4 \\
\hline DiGeorge syndrome & 4 & 9.3 & 1 & 6.7 & 5 & 8.6 \\
\hline Total & 43 & 100 & 15 & 100 & 58 & 100 \\
\hline Missing data (type description) & 0 & & 1 & & 1 & \\
\hline Grand total & 43 & & 16 & & 59 & \\
\hline \multirow[t]{2}{*}{ Defective PTH action } & \multicolumn{2}{|c|}{$\begin{array}{l}\text { Under } 18 \text { at onset of } \\
\text { disease }\end{array}$} & \multicolumn{2}{|c|}{$\begin{array}{l}\text { Over/equal to } 18 \text { at onset } \\
\text { of disease }\end{array}$} & \multicolumn{2}{|l|}{ Total } \\
\hline & $N$ & $\%$ & $N$ & $\%$ & $N$ & $\%$ \\
\hline Pseudohypoparathyroidism type 1a & 8 & 66.7 & 4 & 28.6 & 12 & 46.2 \\
\hline Pseudohypoparathyroidism type $1 \mathrm{~b}$ & 4 & 33.3 & 10 & 71.4 & 14 & 53.8 \\
\hline Total & 12 & 100 & 14 & 100 & 26 & 100 \\
\hline Missing data (type description) & 2 & & 0 & & 2 & \\
\hline Grand total & 14 & & 14 & & 28 & \\
\hline
\end{tabular}

$* N$ number of subjects

over $300 \mathrm{mg} / 24 \mathrm{~h}$ (mean value: $253 \pm 182.14 \mathrm{mg} / 24 \mathrm{~h}$ ). In adult female patients (total number: 368 ), this parameter was available for $47.3 \%$ ( $N: 174)$; among these, in $38.9 \%$ it was over $250 \mathrm{mg} / 24 \mathrm{~h}$ (mean value: $236.3 \pm 162.53$ ). PHP adult patients did not show hypercalciuria (this exam was available for $47 \%$ of patients, $N: 8$ ).

Only nine adult patients (4.4\% of patients observed, $N$ : 206) with chronic hypoparathyroidism had hypocalcemia, hyperphosphatemia and hypercalciuria simultaneously, and none among patients with PHP. The renal function data (creatinine and eGFR levels) were not collected at baseline evaluation, but will be monitored in future prospective studies.

The level of 25 hydroxyvitamin D was available for $44.4 \%$ of the patients with chronic hypoparathyroidism $(N$ : 226); among these, $42.3 \%$ were within range (30-100 ng/dl), and under $30 \mathrm{ng} / \mathrm{dl}$ in $57.7 \%$. This parameter in PHP patients was available for $42.8 \%(N: 12)$, and among these was within range in $25 \%$, and under $30 \mathrm{ng} / \mathrm{dl}$ in $75 \%$.

In adult patients with chronic hypoparathyroidism, the mean value of bone turnover markers, performed in $20 \%$ of patients, showed a normal-low bone turnover (bone 


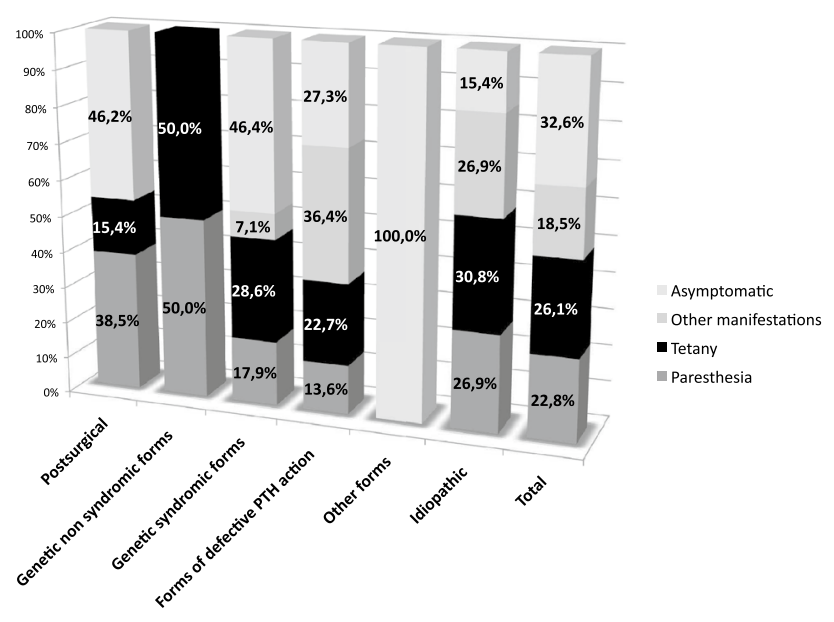

Fig. 4 Clinical manifestations at onset of all main types of hypoparathyroidism ( $<18$ years of age)

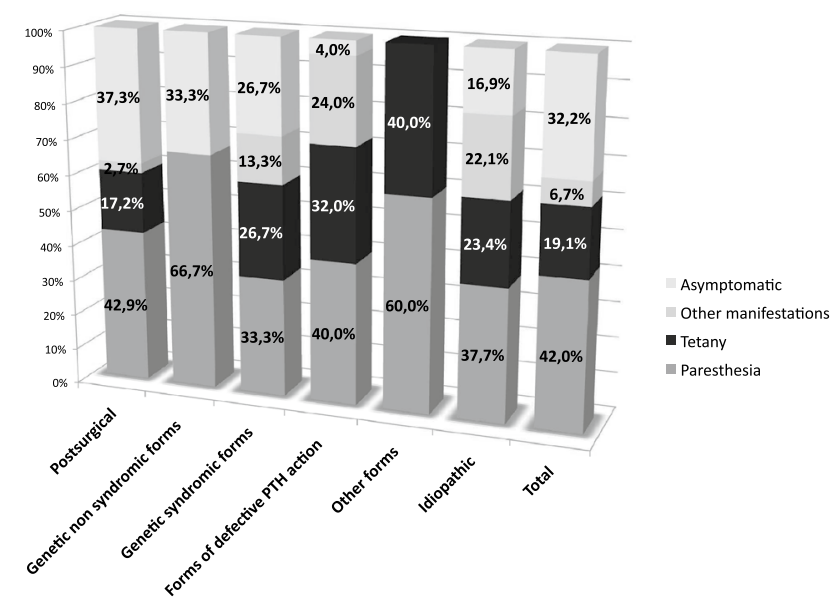

Fig. 5 Clinical manifestations at onset of all main types of hypoparathyroidism ( $\geq 18$ years of age)

alkaline phosphatase: mean value in adults $11.45 \mathrm{mcg} / \mathrm{l}$, range 4-22.5; urinary deoxypyridinoline: mean value in adults $5.27 \mathrm{nmol} / \mathrm{mmol}$, range 3-7.4). Bone turnover markers were not measured in patients with PHP.

\section{Bone Status}

Regarding instrumental exams, dual-energy X-ray absorptiometry (DXA) to measure bone mineral density (BMD) was performed only in 180 patients (35.4\%), among these, 171 adults. This analysis is thus limited by the low number of patients who underwent this type of instrumental exam; however, the mean values of BMD in this population of patients showed high-normal values, except for a few cases (mean value in adult patients: lumbar BMD 1.047 \pm 0.236 , $T$ score: $-0.2 \pm 2, Z$ score: $0.4 \pm 1.9$; total femur BMD
$1.010 \pm 0.570, T$ score: $-0.3 \pm 1.2, Z$ score: $0.5 \pm 1.2$; and femur neck $0.853 \pm 0.302, T$ score $-0.8 \pm 1.8, Z$ score $0.1 \pm 1.2$ ). In PHP patients, DXA was performed in 16 adult subjects (57.1\%), and the mean values of BMD were: lumbar BMD $1.307 \pm 0.146, T$ score: $0.1 \pm 1.6, Z$ score: $-0.5 \pm 1.1$; total femur $T$ score: $-1.1 \pm 0.1, Z$ score: $-0.9 \pm 0.0$, and femur neck $T$ score $-0.9 \pm 0.3, Z$ score $-1.10 \pm 0.1$.

Three patients with chronic hypoparathyroidism having lumbar $T$ score values $<-2.5$ were identified (mean -2.9 , min: -2.8 , max: -3.1 ), and $T$ score femur equal to -1.5 . All three cases are subjects $>50$ years of age (mean age 61), two female and one male.

\section{Others Instrumental Exams}

In $23.4 \%$ of patients with chronic hypoparathyroidism, further ultrasound or radiological examinations were performed, such as echocardiogram (8.5\% of total), audiometry ( $0.4 \%$ of total), cranial computed tomography (CT) $(7.3 \%$ of total), and X-ray examination of the skeleton (7.3\% of total). In PHP patients, echocardiogram was performed in $21.4 \%$, audiometry in $7.1 \%$, and CT in $25 \%$ of total. Among 44 patients with chronic hypoparathyroidism and 6 with PHP, who underwent echocardiogram, cardiac hypertrophy was reported in 4 adult patients ( 2 patients with postsurgical hypoparathyroidism, and 2 with PHP). The average duration of hypoparathyroidism was variable (mean duration: 10 years, min: 1 year and max: 20 years). All patients were treated with calcium and calcitriol with average dosages of, respectively, $1.8 \mathrm{~g} /$ day (min: $1 \mathrm{~g} /$ day, max: $2 \mathrm{~g} /$ day) and 0.62 $\mathrm{mcg} / \mathrm{day}$ (min: $0.25 \mathrm{mcg} / \mathrm{day}$, max: $1 \mathrm{mcg} / \mathrm{day}$ ). Moreover, five patients showed valvular calcifications (four cases of postsurgical hypoparathyroidism, and one case of hypoparathyroidism associated with APS1). The average duration of hypoparathyroidism was variable (mean duration: 15 years, min: 1 year and max: 38 years). All patients were treated with calcium and calcitriol with average dosages of, respectively, $2.4 \mathrm{~g} /$ day (min: $1.5 \mathrm{~g} /$ day, max: $4 \mathrm{~g} /$ day) and 0.75 $\mathrm{mcg} / \mathrm{day}$ (min: $0.5 \mathrm{mcg} / \mathrm{day}$, max: $1 \mathrm{mcg} /$ day). Among 44 patients, of which 7 with PHP, who underwent cranial CT, $33(75 \%)$ showed cerebral calcifications; of these, 11 were affected by genetic forms of hypoparathyroidism, 20 by idiopathic forms, and the remaining by postsurgical forms. A case of tetralogy of Fallot was identified in a patient with DiGeorge syndrome.

\section{Genetic Data}

Genetic analysis was performed in 80 subjects only, and a genetic diagnosis was established in only 65 cases. Thirtyfour were diagnosed with mutations of AIRE gene (APS1), 20 with mutations of GNAS (guanine nucleotide-binding protein, alpha-stimulating) gene (PHP type $1 \mathrm{a}$ and $1 \mathrm{~b}$ ), 5 
with 22q11.2 microdeletions (DiGeorge syndrome), and 3 with mutations of CaSR (Calcium-Sensing Receptor) gene (autosomal-dominant hypocalcemia with hypercalciuria type 1). Anti-CaSR antibodies were not tested in any APS1 cases.

\section{Treatment}

Regarding chronic hypoparathyroidism treatment at baseline evaluation, most patients were treated with calcium and vitamin D supplements (metabolites and analogs) and a few cases (adult patients) treated with rhPTH (1-34), in particular, 10 subjects ( 7 with postsurgical hypoparathyroidism, 1 with APS1, and 2 with idiopathic hypoparathyroidism).

In adult patients with chronic hypoparathyroidism, the mean doses of calcium and calcitriol supplementation (considering, respectively, $8 \%$ and $10.7 \%$ of missing data) were, respectively, $1.62 \pm 0.98 \mathrm{~g} /$ day (mode: $1 \mathrm{~g} /$ day) and $0.69 \pm 0.48 \mathrm{mcg} / \mathrm{day}$ (mode: $0.50 \mathrm{mcg} /$ day), of cholecalciferol (taken only by $16.9 \%$ of total number of adult patients) $1668 \pm 1343.3 \mathrm{UI} /$ day. Hydrochlorothiazide, a thiazide diuretic, was used by 11 cases (2\%); of these, 3 were affected by idiopathic forms and 8 by postsurgical forms. Of note, 74 patients $(16.1 \%)$ needed large amounts of calcium supplementation $(>2.5 \mathrm{~g})$ and $17(3.7 \%)$ needed large amounts of calcitriol supplementation ( $>1.5 \mathrm{mcg} /$ day), and only 3 patients $(0.7 \%)$ took these doses of both drugs.

In adult PHP patients, the mean doses of calcium and calcitriol supplementation (considering, respectively, $17.6 \%$ and $11.8 \%$ of missing data) were, respectively, $1.83 \pm 0.98 \mathrm{~g} /$ day (mode: $2 \mathrm{~g} /$ day) and $0.70 \pm 0.28 \mathrm{mcg} /$ day (mode: 0.50 $\mathrm{mcg} /$ day), of cholecalciferol [taken by 14 cases $(2220 \pm 860$ UI/day)]. Of note, two patients needed large amounts of calcium supplementation $(>2.5 \mathrm{~g})$.

\section{Discussion}

Data relating to the epidemiology and clinical-therapeutic characterization of chronic hypoparathyroidism are not yet well defined, especially in Italy, where only one study has investigated the prevalence of hypoparathyroidism among hospitalized patients through a retrospective register-based study [13].

Our observational retrospective study collected 537 clinical cases with chronic hypoparathyroidism, of which 28 with PHP, referred to 20 specialized medical and surgical centers, including a comprehensive description of demographic, etiological, clinical and therapeutic data. The centers involved are limited in number, but evenly distributed throughout the Italian territory (including 11 regions), and characterized by long-term expertise on this rare endocrine disorder.

The analysis of this database confirmed that most patients affected by chronic hypoparathyroidism were female, and the most common form of this disease was postsurgical hypoparathyroidism, as described in literature [11, 13]. Among the cases of postsurgical hypoparathyroidism, most cases occurred after a total thyroidectomy, followed by parathyroidectomy with removal of the parathyroid glands, confirming, also in this case, data in literature [10]. Our data also revealed a further subdivision, not performed in other studies, between the subjects under and over/equal to 18 years, showing that most causes of chronic hypoparathyroidism described under 18 years of age were syndromic forms of genetic hypoparathyroidism, in particular APS, while the most frequent cause of chronic hypoparathyroidism described over/equal to 18 years of age was neck surgery, in particular post-total thyroidectomy. Moreover, in our database several cases of idiopathic forms were identified, probably due to the fact that genetic investigations and instrumental exams were carried out only in a fraction of the probands. Therefore, an assessment of the mutations associated with hypoparathyroidism should be performed more frequently and, in the future, research for new mutations causing hypoparathyroidism should be implemented.

In a few cases, a DXA exam was carried out at baseline evaluation; among those analyzed, a high-normal bone mass density than age- and gender-matched controls was described, in accordance with a low bone turnover profile typical of the disease $[1,17,18]$. Recent studies have described that the higher BMD in hypoparathyroidism is due in large part to an increase in bone tissue volume rather than an increase in amount of mineral within the tissue, and that there is an increase in cancellous bone volume and trabecular thickness, and a higher trabecular number and trabecular connectivity in comparison with matched control subjects $[1,19]$. However, further studies are necessary for a better understanding of bone pathophysiology and bone quality in hypoparathyroidism, as well as the biomechanical and metabolic effects of a disease which, until a few years ago, were poorly studied.

Regarding cardiovascular complications, only a small percentage of patients were examined with echocardiogram. Cardiological monitoring of these patients will allow us to increase our knowledge of the natural history of this pathology, and the impact of the diseases and its therapies from this point of view.

Basal ganglia calcification is a typical complication of chronic hypoparathyroidism [1]. In our cohort, the prevalence of cerebral calcifications was high at $75 \%$ of the small subset, with imaging to review at baseline evaluation, as reported in another study [20]. Other studies described a lower percentage; however, all analyses were conducted on a small cohort [17, 21].

Regarding biochemical exams, overall, the data collected at the baseline evaluation showed numerous cases with values above or below the reference range of 
corrected serum calcium, serum phosphorus, and $24 \mathrm{~h}$ urinary calcium, in accordance with the known difficulty to maintain these levels within the range of normality [1, $14,20,22]$. Of note, only $18.9 \%$ of patients with chronic hypoparathyroidism had serum-corrected calcium between 8.0 and $8.5 \mathrm{mg} / \mathrm{dl}$, and $13.6 \%$ in PHP patients. An analysis of the biochemical tests performed over a long period of follow-up could provide a broader assessment of the control of calcium-phosphate homeostasis. However, the evaluation of biochemical exams at baseline visit, being evaluated on a large number of patients, although in the presence of missing data, can give an indication of the challenges associated with the control of calcium-phosphate homeostasis. Sub-optimal serum calcium levels can cause paresthesias, prolonged QTc on ECG (electrocardiogram), impaired renal function, brain fog, dizziness, and reduced quality of life. On the other hand, an elevated serum calcium-phosphate product, albeit found in a small percentage of our patients, can lead, over the years, to soft tissue extraskeletal calcifications, typically seen in the brain (especially basal ganglia calcifications) and in the kidney (stones and nephrocalcinosis) [23, 24].

In more than half of all cases observed, serum 25 hydroxyvitamin D concentration was under $30 \mathrm{ng} / \mathrm{dl}$, and in almost $40 \%$ serum magnesium was low in adult patients evaluated. These two data emphasize the importance of providing an adequate supplementation of cholecalciferol and magnesium in these patients. In particular, 25 hydroxyvitamin D is probably important in several cellular processes, because intracellular hydroxylation to active vitamin D occurs in many different cells, such as bone, gut, and prostate. Moreover, a status of vitamin $\mathrm{D}$ insufficiency can be associated to neuromuscular problems. Therefore, it is reasonable to ensure an adequate vitamin D status also in these patients [14].

Regarding management of chronic hypoparathyroidism at baseline evaluation, in almost all cases, standard treatment options were used (oral calcium, metabolites and vitamin D analogs), except for a few cases treated with rhPTH (1-34), since the conventional treatment was not able to adequately control the disease. Among patients prescribed with standard treatment, a small group required large amounts of calcium and calcitriol. Although this group is a small percentage compared to the total number, it represents a serious therapeutic problem, considering the risk of long-term complications (i.e. nephrolithiasis, nephrocalcinosis, renal impairment, cataracts and cerebral calcifications).

In the future, biochemical exams, as recommended by recent guidelines [14, 15], should be collected and analyzed with longitudinal prospective studies in association with a monitoring of complications, and doses of calcium supplements and vitamin D required, to define the percentage of patients adequately treated, or not, with conventional treatment in accordance with the guidelines $[14,15]$.

\section{Conclusions}

In conclusion, this study represents one of the first largescale assessments of epidemiology of chronic hypoparathyroidism at expert medical-surgical centers, distributed homogeneously in the Italian territory. The differences in the number of cases in each center can be linked to the diversity of type and location of hospitals.

However, the study has some limitations, such as the absence of some clinical data, especially given that the analysis was retrospective in nature. In addition, patients with mild presentations could not be referred to these specialized centers, resulting in an underestimation of the true burden of the disease at each center. Nevertheless, the project introduces the possibility of a future large-scale prospective analysis that would help define the exact prevalence/incidence of chronic hypoparathyroidism in Italy, associated with a complete clinical and therapeutic characterization. Considering the prevalence reported in the cited Cipriani et al. paper [13], although focused on cases of postsurgical hypoparathyroidism and, therefore underestimated, the group of patients with hypoparathyroidism of our database could represent about 1/5 of Italian hypoparathyroidism patients. Therefore, the registry will have to be expanded in the future. In this regard, this database is already the basis for new projects, aimed at the creation of new registries. Moreover, a longitudinal prospective observational nationwide study, performed over a long period, could also provide information on the natural history, short- and long-term complications, and healthcare costs of this important endocrinological chronic disease and related treatment. These analyses will also be useful in consideration of the new possible hormone replacement therapy with rhPTH (1-84), which will soon be available also in Italy [25, 26]. Of note, future prospective studies could investigate bone fracture risks and ocular, cardiovascular, renal, neurological, and psychological complications in these patients, and the results will provide further guidance in this regard.

Acknowledgements SHIRE, pharmaceutical company.

Data Availability All data analyzed during this study are included in this published article.

Author's Contributions MLB was the coordinator of the project and designed the study. All authors contributed to the collection of clinical data, and the paper was drafted by GM, LC and MLB. SP was responsible for statistical analysis of the data. All authors revised the paper critically for intellectual content and approved the final version. All authors agree to be accountable for the work and to ensure that any questions relating to the accuracy and integrity of the paper are investigated and properly resolved.

Funding This study was funded by Shire, a pharmaceutical company. 


\section{Compliance with Ethical Standards}

Conflict of interest G. Mantovani: member of advisory boards for Shire; C. Marcocci: consultant for Shire Italia. M.L. Brandi: is a consultant for Alexion, Bruno Farmaceutici, Shire, Servier, Kyowa Kirin; academic grants and/or speaker: Abiogen, Alexion, Amgen, Bruno Farmaceutici, Eli Lilly, Kyowa Kirin, MSD, NPS, Servier, Shire, SPA; she has received honoraria from: Amgen, Bruno Farmaceutici, Kyowa Kirin. The other authors declare that they have no competing interests that could be perceived as prejudicing the impartiality of the research reported.

Consent for Publication The consent to the publication of the data was obtained.

Ethical Approval The study was approved by the Institutional Review Board (Comitato Etico Area Vasta Centro, AUOC, Florence, Italy); Number: 10641_oss.

Human and Animal Rights and Informed Consent All procedures performed in studies involving human participants were in accordance with the ethical standards of the institutional and/or national research committee and with the 1964 Helsinki declaration and its later amendments or comparable ethical standards. For this type of study formal consent is not required.

\section{References}

1. Bilezikian JP, Khan A, Potts JT Jr, Brandi ML, Clarke BL, Shoback D, Jüppner H, D’Amour P, Fox J, Rejnmark L, Mosekilde L, Rubin MR, Dempster D, Gafni R, Collins MT, Sliney J, Sanders J (2011) Hypoparathyroidism in the adult: epidemiology, diagnosis, pathophysiology, target-organ involvement, treatment, and challenges for future research. J Bone Miner Res 10:2317-2337

2. Shoback D (2008) Clinical practice. Hypoparathyroidism. N Engl J Med 4:391-403

3. Marcucci G, Cianferotti L, Beck-Peccoz P, Capezzone M, Cetani F, Colao A, Davì MV, degli Uberti E, Del Prato S, Elisei R, Faggiano A, Ferone D, Foresta C, Fugazzola L, Ghigo E, Giacchetti G, Giorgino F, Lenzi A, Malandrino P, Mannelli M, Marcocci C, Masi L, Pacini F, Opocher G, Radicioni A, Tonacchera M, Vigneri R, Zatelli MC, Brandi ML (2015) Rare diseases in clinical endocrinology: a taxonomic classification system. J Endocrinol Invest 2:193-259

4. Marx SJ (2000) Hyperparathyroid and hypoparathyroid disorders. N Engl J Med 25:1863-1875

5. Thomusch O, Machens A, Sekulla C, Ukkat J, Brauckhoff M, Dralle H (2003) The impact of surgical technique on postoperative hypoparathyroidism in bilateral thyroid surgery: a multivariate analysis of 5846 consecutive patients. Surgery 2:180-185

6. Zarnegar R, Brunaud L, Clark OH (2003) Prevention, evaluation, and management of complications following thyroidectomy for thyroid carcinoma. Endocrinol Metab Clin North Am 2:483-502

7. Page C, Strunski V (2007) Parathyroid risk in total thyroidectomy for bilateral, benign, multinodular goitre: report of 351 surgical cases. J Laryngol Otol 3:237-241

8. Underbjerg L, Sikjaer T, Mosekilde L, Rejnmark L (2015) The epidemiology of nonsurgical hypoparathyroidism in Denmark: a nationwide case finding study. J Bone Miner Res 9:1738-1744

9. Underbjerg L, Sikjaer T, Mosekilde L, Rejnmark L (2013) Cardiovascular and renal complications to postsurgical hypoparathyroidism: a Danish nationwide controlled historic follow-up study. J Bone Miner Res 11:2277-2285

10. Underbjerg L, Sikjaer T, Mosekilde L, Rejnmark L (2014) Postsurgical hypoparathyroidism-risk of fractures, psychiatric diseases, cancer, cataract, and infections. J Bone Miner Res 11:2504-2510

11. Powers J, Joy K, Ruscio A, Lagast H (2013) Prevalence and incidence of hypoparathyroidism in the United States using a large claims database. J Bone Miner Res 12:2570-2576

12. Astor MC, Løvås K, Debowska A, Eriksen EF, Evang JA, Fossum C, Fougner KJ, Holte SE, Lima K, Moe RB, Myhre AG, Kemp EH, Nedrebø BG, Svartberg J, Husebye ES (2016) Epidemiology and health-related quality of life in hypoparathyroidism in Norway. J Clin Endocrinol Metab 8:3045-3053

13. Cipriani C, Pepe J, Biamonte F, Manai R, Biondi P, Nieddu L, Cianferotti L, Brandi ML, Minisola S (2017) The Epidemiology of hypoparathyroidism in Italy: an 8-year register-based study. Calcif Tissue Int 3:278-285

14. Brandi ML, Bilezikian JP, Shoback D, Bouillon R, Clarke BL, Thakker RV, Khan AA, Potts JT Jr (2016) Management of hypoparathyroidism: summary statement and guidelines. J Clin Endocrinol Metab 6:2273-2283

15. Bollerslev J, Rejnmark L, Marcocci C, Shoback DM, Sitges-Serra A, van Biesen W, Dekkers OM; European Society of Endocrinology (2015) European Society of Endocrinology Clinical Guideline: treatment of chronic hypoparathyroidism in adults. Eur $\mathbf{J}$ Endocrinol 2:G1-20

16. Natpara package insert. http://www.accessdata.fda.gov/drugsatfda _docs/label/2015/125511s000lbl.pdf. Accessed 22 june 2015

17. Rubin MR, Dempster DW, Zhou H et al (2008) Dynamic and structural properties of the skeleton in hypoparathyroidism. J Bone Miner Res 23:2018-2024

18. Rubin MR, Dempster DW, Kohler T et al (2010) Three dimensional cancellous bone structure in hypoparathyroidism. Bone 46:190-195

19. Rubin MR, Dempster DW, Kohler T, Stauber M, Zhou H, Shane E, Nickolas T, Stein E, Sliney J Jr, Silverberg SJ, Bilezikian JP, Müller R (2010) Three dimensional cancellous bone structure in hypoparathyroidism. Bone 1:190-195

20. Mitchell DM, Regan S, Cooley MR, Lauter KB, Vrla MC, Becker CB, Burnett-Bowie SA, Mannstadt M (2012) Long-term followup of patients with hypoparathyroidism. J Clin Endocrinol Metab 97(12):4507-4514

21. Goswami R, Sharma R, Sreenivas V, Gupta N, Ganapathy A, Das S (2012) Prevalence and progression of basal ganglia calcification and its pathogenic mechanism in patients with idiopathic hypoparathyroidism. ClinEndocrinol (Oxf) 2:200-206

22. Arlt W, Fremerey C, Callies F, Reincke M, Schneider P, Timmermann W, Allolio B (2002) Well-being, mood and calcium homeostasis in patients with hypoparathyroidism receiving standard treatment with calcium and vitamin D. Eur J Endocrinol $2: 215-222$

23. De Sanctis V, Soliman A, Fiscina B (2012) Hypoparathyroidism: from diagnosis to treatment. Curr Opin Endocrinol Diabetes Obes 6:435-442

24. Mannstadt MM, Mitchell DM (2015) Clinical manifestations of hypoparathyroidism. In: Bilezikian JP, Marcus R, Levine MA, Marcocci C, Silverberg SJ, Potts JT Jr (eds) The parathyroids. Academic Pressp, London, pp 761-770

25. Marcucci G, Della Pepa G, Brandi ML (2016) Natpara for the treatment of hypoparathyroidism. Expert Opin Biol Ther 16:1417-1424

26. Marcucci G, Della Pepa G, Brandi ML (2017) Drug safety evaluation of parathyroid hormone for hypocalcemia in patients with hypoparathyroidism. Expert Opin Drug Saf 16:617-625 


\section{Affiliations}

Gemma Marcucci ${ }^{1}$. Luisella Cianferotti ${ }^{1}$. Simone Parri ${ }^{1}$. Paola Altieri ${ }^{2} \cdot$ Emanuela Arvat $^{3} \cdot$ Salvatore Benvenga $^{4}$. Corrado Betterle ${ }^{5}$. Marta Bondanelli ${ }^{6}$. Marco Boscaro ${ }^{5}$. Valentina Camozzi ${ }^{5}$. Grazia Maria Centaro ${ }^{7}$. Filomena Cetani ${ }^{8} \cdot$ Iacopo Chiodini $^{9} \cdot$ Anna Ciampolillo $^{10}$. Annamaria Colao ${ }^{11}$. Sabrina Corbetta ${ }^{12}$. Maria Laura De Feo ${ }^{13}$. Ettore degli Uberti ${ }^{6}$. Antongiulio Faggiano ${ }^{14}$. Rachele Fornari ${ }^{15}$. Achille Lucio Gaspari ${ }^{16}$. Francesco Giorgino ${ }^{10} \cdot$ Valeria Giuliani $^{13} \cdot$ Maurizio lacobone $^{17}$. Nadia Innaro ${ }^{18}$. Olga Lamacchia ${ }^{19} \cdot$ Andrea Lenzi $^{15}$. Giovanna Mantovani ${ }^{9}$. Claudio Marcocci ${ }^{20}$. Laura Masi $^{21}$ - Silvia Migliaccio ${ }^{22}$. Serena Palmieri ${ }^{9} \cdot$ Renato Pasquali $^{2}$. Giuliano Perigli ${ }^{23}$. Valentina Piccini ${ }^{13}$. Elisabetta Romagnoli ${ }^{15} \cdot$ Rosaria Maddalena Ruggeri $^{4} \cdot$ Francesco Rulli $^{24}$. Maria Teresa Samà ${ }^{3}$. Giuseppe Tomaino ${ }^{17}$. Francesco Trimarchi $^{25} \cdot$ Maria Chiara Zatelli $^{6} \cdot$ Maria Luisa Brandi $^{1}$

Gemma Marcucci

gemma.marcucci@unifi.it

Luisella Cianferotti

luisella.cianferotti@unifi.it

Simone Parri

simone@parri.it

Paola Altieri

paola.altieri@unibo.it

Emanuela Arvat

emanuela.arvat@unito.it

Salvatore Benvenga

s.benvenga@live.it

Corrado Betterle

corrado.betterle@unipd.it

Marta Bondanelli

bndmrt@unife.it

Marco Boscaro

marco.boscaro@unipd.it

Valentina Camozzi

valentina.camozzi@unipd.it

Grazia Maria Centaro

grazia.centaro@gmail.com

Filomena Cetani

cetani@endoc.med.unipi.it

Iacopo Chiodini

iacopo.chiodini@policlinico.mi.it

Anna Ciampolillo

anna.ciampolillo@uniba.it

Annamaria Colao

colao@unina.it

Sabrina Corbetta

sabrina.corbetta@unimi.it

Maria Laura De Feo

marialaura.defeo@uslcentro.toscana.it

Ettore degli Uberti

dut@unife.it

Antongiulio Faggiano

afaggian@unina.it

Rachele Fornari

fornari.rachele@gmail.com

Achille Lucio Gaspari

gaspari@med.uniroma2.it
Francesco Giorgino

francesco.giorgino@uniba.it

Valeria Giuliani

valeria.giuliani@uslcentro.toscana.it

Maurizio Iacobone

maurizio.iacobone@unipd.it

Nadia Innaro

nadia.innaro@tiscali.it

Olga Lamacchia

olga.lamacchia@unifg.it

Andrea Lenzi

andrea.lenzi@uniroma1.it

Giovanna Mantovani

giovanna.mantovani@unimi.it

Claudio Marcocci

claudio.marcocci@med.unipi.it

Laura Masi

laura.masi@aouc.unifi.it

Silvia Migliaccio

silvia.migliaccio@uniroma4.it

Serena Palmieri

serena.palmieri@hotmail.it

Renato Pasquali

renato.pasquali@unibo.it

Giuliano Perigli

giuliano.perigli@unifi.it

Valentina Piccini

valentina.piccini@uslcentro.toscana.it

Elisabetta Romagnoli

elisabetta.romagnoli@uniroma1.it

Rosaria Maddalena Ruggeri

rmruggeri@unime.it

Francesco Rulli

rulli@uniroma2.it

Maria Teresa Samà

marysama@tiscali.it

Giuseppe Tomaino

giuseppe_tomaino@me.com

Francesco Trimarchi

francesco.trimarchi@unime.it

Maria Chiara Zatelli

ztlmch@unife.it 
1 Bone Metabolic Diseases Unit, Department of Surgery and Translational Medicine, University of Florence, Florence, Italy

2 Division of Endocrinology, S. Orsola-Malpighi Hospital, University Alma Mater Studiorum of Bologna, Bologna, Italy

3 Oncological Endocrinology Unit, Department of Medical Sciences, University of Turin, Turin, Italy

4 Department of Clinical and Experimental Medicine, University of Messina School of Medicine, Messina, Italy

5 Department of Medicine (DIMED)-Endocrinology, University of Padua, Padua, Italy

6 Section of Endocrinology \& Internal Medicine, Department of Medical Sciences, University of Ferrara, Ferrara, Italy

7 Internal Medicine Hospital, Monsignor Dimiccoli, Barletta, Italy

8 Endocrine Unit 2, University Hospital of Pisa, Pisa, Italy

9 Endocrinology Unit, Fondazione IRCCS Cà Granada Ospedale Maggiore Policlinico, Department of Clinical Sciences and Community Health, University of Milan, Milan, Italy

10 Section of Internal Medicine, Endocrinology, Andrology and Metabolic Diseases, Department of Emergency and Organ Transplantation, University of Bari Aldo Moro, Bari, Italy

11 Department of Clinical Medicine and Surgery, Federico II di Naples University, Naples, Italy

12 Endocrinology Unit, Department of Biomedical Sciences for Health, University of Milan, IRCCS Istituto Ortopedico Galeazzi, Milan, Italy

13 Endocrinology and Diabetology Unit UOSD, Hospital USL 11, Empoli, Italy
14 Thyroid and Parathyroid Surgery Unit, Istituto Nazionale per lo studio e la cura dei tumori "Fondazione G. Pascale" - IRCCS, Naples, Italy

15 Department Experimental Medicine, Section Medical Pathophysiology, Endocrinology and Nutrition, University "Sapienza" of Rome, Rome, Italy

16 Department of Surgery, University Hospital of Tor Vergata, Rome, Italy

17 Endocrine Surgery Unit, Department of Surgery, Oncology and Gastroenterology, University of Padua, Padua, Italy

18 Endocrine Surgery UOC, University College of Catanzaro/Policlinico universitario, Catanzaro, Italy

19 Unit of Endocrinology and Metabolic Diseases, Department of Medical and Surgical Sciences, University of Foggia, Foggia, Italy

20 Department of Endocrinology and Metabolism, University of Pisa, Pisa, Italy

21 Bone Metabolic Diseases Unit, University Hospital of Florence, Florence, Italy

22 Unit of Endocrinology, Department of Movement, Human and Health Sciences, University "Foro Italico" of Rome, Rome, Italy

23 Department of Surgery and Translational Medicine, University of Florence, Florence, Italy

24 Department of Surgical Sciences, Catholic University "Our Lady of Good Counsel", Tirana, Albania

25 Accademia Peloritana dei Pericolanti, University of Messina, Messina, Italy 